IAU Colloguium 164: Radio Emission from Galactic and Extragalactic Compact Sources

ASP Conference Series, Vol. 144,1998

J. A. Zensus, G. B. Taylor, \& J. M. Wrobel (eds.)

\title{
X-Band VLBA Polarization of 3C 287
}

D. Dallacasa

Istituto di Radioastronomia, CNR, Bologna, Italy

R. T. Schilizzi

Joint Institute for VLBI in Europe, Dwingeloo, The Netherlands

Nan Rendong

Beijing Radio Observatory, Beijing, China

Abstract. VLBA polarimetric observations at 8.15 and $8.54 \mathrm{GHz}$ of the quasar $3 \mathrm{C} 287$ are presented and discussed. The source structure is complex, and the magnetic field geometry does not help in the interpretation of the source.

\section{Introduction}

Compact steep-spectrum (CSS) radio sources are intrinsically small (subgalactic size) and powerful objects. Their polarized emission is generally weak, with a few exceptions among CSS quasars. The models either propose that CSS are "frustrated sources", confined to the nuclear region by a very dense interstellar medium (van Breugel, Miley, \& Heckman 1984), or that they are young and will grow large with age, decreasing the radio luminosity by about one order of magnitude (Fanti et al. 1995, Readhead et al. 1996).

In particular, the CSS quasar 3C 287 (1328+254, at redshift $\mathrm{z}=1.055)$ was known to posses an arc-like VLBI morphology possibly indicative of a core-jet structure (Fanti et al. 1989), while its polarized emission is at a few percent level at $\mathrm{cm}$ wavelengths as derived from single dish measurements. In order to study the polarization properties of this source, we carried out VLBA observations at two frequencies spread across the $\mathrm{X}$-band, aiming to reveal the magnetic field geometry and to detect possible high rotation measure (RM), commonly found among CSS sources.

\section{Results}

The source is well resolved by the present VLBA observations and only about $35 \%$ of the total flux density is accounted for in our images, while almost the whole polarized emission (as measured from single dish data) is detected by the present data.

Our images at both frequencies show the same structure in both total intensity and polarization (see the image at $8.54 \mathrm{GHz}$ in Fig. 1). The earlier interpretation by Fanti et al. (1989) would imply a compact emission with significant flux density in correspondence of their core candidates. However, there is no compact component with flux density exceeding a few $\mathrm{mJy}$ at $\mathrm{X}$ band, since the source is not detected at a few mJy level on the longest VLBA baselines. Even the structure imaged here is completely resolved out on baselines longer than $20 \mathrm{M} \lambda$.

The polarized emission comes from the brightest region in total intensity; we could not exclude further polarized emission from other regions with lower 
surface brightness, where it could not be detected due to $S / N$ reasons. The magnetic field geometry is rather complex since it shows $90^{\circ}$ turns on scales of about 10 milliarseconds, and does not help in the interpretation of the radio source structure.

There is little or no Faraday rotation between the two observing frequencies $(8.15$ and $8.54 \mathrm{GHz})$. The difference between the angles seen at the two bands is consistent with 0 deg on most of the source, although we need another frequency at least, in order to confirm this result.

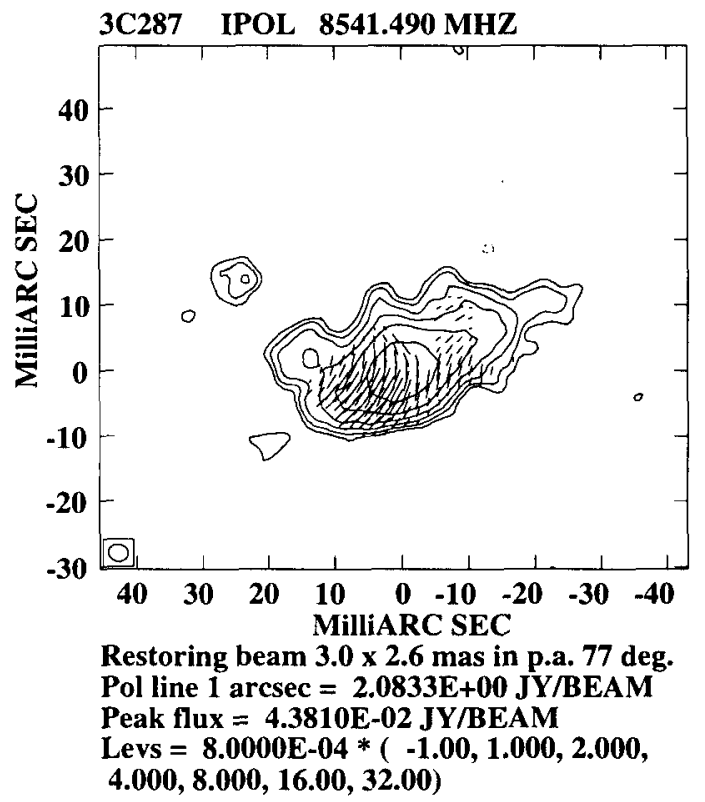

Figure 1. VLBA polarization image of $3 \mathrm{C} 287$. The polarization vectors (E field) are plotted

Acknowledgments. DD acknowledges the organization for partial financial support. The National Radio Astronomy Observatory is a facility of the National Science Foundation, operated under a cooperative agreement by Associated Universities, Inc.

\section{References}

Fanti, C., et al. 1989. $A \& A, 217,44-56$.

Fanti, C., et al. 1995. $A \mathscr{E} A, 302,317-326$.

Readhead, A. C. S., et al. 1996. ApJ, 460, 634-643.

van Breugel, W., Miley, G., \& Heckman, T. 1984. AJ, 89, 5-22. 